\title{
Análisis de Flujos de Potencias Ineficientes Producidos por Convertidores de Potencia
}

\author{
Juan D. Gallego-Gómez, Juan B. Cano-Quintero, y Nicolás Muñoz-Galeano \\ Universidad de Antioquia, Fac. de Ingeniería, Depto Ing. Eléctrica, Grupo de Manejo Eficiente de la Energía \\ - GIMEL, Calle 67 No. 53-108, Oficina 19-437, Medellín-Colombia (e-mail: jdgallego87@yahoo.es, \\ jbcano@gmail.com, nicolasm@udea.edu.co)
}

Recibido Nov. 5, 2013; Aceptado Ene. 2, 2014; Versión final recibida Ene. 20, 2014

\begin{abstract}
Resumen
Este artículo presenta un análisis de los flujos de potencia ineficientes provocados por los convertidores de potencia. En base a la teoría de la potencia del estándar IEEE 1459 (2010) y partiendo de la potencia causada por las corrientes armónicas $\left(D_{e l)}\right)$, se proponen las potencias $D_{e l \_ \text {low }}$ y $D_{e l \_ \text {high }}$ para cuantificar los flujos de potencia ineficiente provocados por los convertidores de potencia. La potencia $D_{e l \_ \text {low }}$ se debe a la existencia de componentes armónicas de corriente de baja frecuencia, mientras que la potencia $D_{\text {el_high }}$ depende del número de pulsos utilizados en la modulación. Se presenta un análisis del intercambio de potencia entre el convertidor, la carga, y los semiconductores. También se incluyen resultados de simulación para un convertidor monofásico controlado mediante la modulación de ancho de pulso senoidal (SPWM, Sinusoidal Pulse Width Modulation) teniendo en cuenta la caída de tensión en los semiconductores de los interruptores de potencia y variando el número de pulsos de la modulación.
\end{abstract}

Palabras clave: ancho de pulso senoidal, SPWM, convertidores de potencia, flujos de potencia

\section{Analysis of Inefficient Power Flows Produced by Power Converters}

\begin{abstract}
This paper presents an analysis of inefficient power flows caused by power converters. Based on the power theory of the IEEE standard 1459 (2010) and starting from the power caused by harmonic currents $\left(D_{e l}\right)$, the power $D_{\text {el_low }}$ and $D_{\text {el_high }}$ are proposed for quantifying the inefficient power flows caused by power converters. The power $D_{e l}$ low is because the existence of low-frequency harmonic currents, while the power $D_{e l \_ \text {high }}$ depends on the number of pulses used in the modulation. An exchange power analysis between the converter, the load, and the semiconductors is presented. There are also included simulation results for a single-phase converter controlled by a Sinusoidal Pulse Width Modulation (SPWM) taking into account the voltage drop in the semiconductors of power switches and changing the number of pulses in the modulation.
\end{abstract}

Keywords: sinusoidal pulse width, SPWM, power converters, power flows 


\section{INTRODUCCIÓN}

Los convertidores de potencia sirven para transformar el voltaje, la frecuencia, la potencia, y el número de fases de acuerdo a los requerimientos de las cargas eléctricas. Los convertidores están compuestos por interruptores de potencia controlados mediante técnicas de modulación. Las técnicas de modulación más usadas son la modulación senoidal de ancho de pulso (Senoidal Pulse Width Modulation, SPWM) y la modulación de espacio vectorial (Space Vector Modulation, SVM) Rus et al. (2010), aunque existen múltiples modulaciones derivadas de las modulaciones SPWM y SVM. Las principales aplicaciones de los convertidores de potencia son: variadores de frecuencia Kojabadi (2011), compensadores activos Orts et al. (2010), sistemas de alimentación ininterrumpida Komurcugil (2012), convertidores de una fase a tres fases Kim et al. (2002) y Jacobina et al. (2010), entre otras.

Las señales de salida de un convertidor deben satisfacer sus requisitos de diseño. En el caso de los variadores de velocidad, deben de entregar una tensión senoidal con bajo contenido de armónicos Kojabadi (2011). En el caso de los compensadores activos, deben entregar las corrientes ineficientes (corrientes reactivas, armónicas, y de desequilibrio) que demandan las cargas eléctricas Orts et al. (2010). En cualquier caso, los convertidores no deben generar nuevas componentes de corriente o voltaje armónico. Debido al carácter discreto de la conmutación de los interruptores, los convertidores siempre inyectan a la carga o a la red de suministro armónicos de corriente, unos en mayor medida que otros dependiendo de la estrategia de modulación y de los filtros que tenga en su salida. Tal inyección de corriente causa flujos de potencia ineficiente entre el convertidor, las cargas, y la red de suministro. El análisis de los flujos de potencia ineficientes producidos por el convertidor es el tema de trabajo en este artículo.

Los flujos de potencia en los sistemas eléctricos se pueden estudiar mediante el uso de la teoría de la potencia instantánea Muñoz et al. (2011) y Seguí et al. (2007), mediante esta teoría se puede determinar si las potencias de los sistemas eléctricos son eficientes o ineficientes. Además, los flujos de potencia se pueden cuantificar usando el estándar IEEE 1459 (2010). El estándar IEEE 1459 (2010) es la última teoría de potencia eléctrica reconocida e incluye una descomposición de las magnitudes de potencia presentes en los sistemas eléctricos. En este artículo, se explican los flujos de potencia de los convertidores enfatizando en el flujo provocado por las corrientes armónicas $\left(D_{e I}\right)$. Basados en el estándar IEEE 1459 (2010) se propone a $D_{\text {eI_low }}$ y $D_{\text {eI_high }}$ como términos de potencia para cuantificar los flujos causados por las corrientes armónicas generadas por el convertidor. Hasta el momento no existe en la literatura técnica ningún artículo que diferencie las causas de los flujos de potencia en los convertidores de potencia. Lo que usualmente hacen es determinar de forma global la distorsión armónica de corriente. En este artículo se diferencian los flujos de potencia del convertidor, se cuantifican y se establecen sus causas. Los convertidores pueden ser monofásicos o trifásicos según la aplicación. En este artículo se utiliza el convertidor monofásico para facilitar la explicación, un análisis similar puede hacerse para convertidores trifásicos.

El artículo se divide en las siguientes secciones. La sección 1 resume los términos de potencia del estándar IEEE 1459 (2010) para sistemas monofásicos, también incluye la descomposición de potencia propuesta para cuantificar los flujos de potencia provocados por el convertidor. La sección 2 presenta las generalidades de los convertidores de potencia y la modulación SPWM. La sección 3 incluye el análisis de los flujos de potencia ineficientes causados por las caídas de tensión en los semiconductores del convertidor. La sección 4 recoge los resultados de simulación. Y finalmente la sección 5 presenta las conclusiones.

\section{CUANTIFICACIÓN DE LAS POTENCIAS DEL ESTANDAR IEEE 1459}

El estándar IEEE 1459 (2010) es la norma en la que se encuentran las definiciones para la cuantificación y medida de las potencias eléctricas bajo condiciones lineales, no lineales, equilibradas y no equilibradas. El estándar IEEE 1459 (2010) reúne el conocimiento desarrollado desde 1920 incluyendo la introducción en los sistemas eléctricos de nuevas cargas y fuentes de potencia. El estándar IEEE 1459 (2010) es la última teoría de potencia reconocida por la comunidad científica y presenta una elegante descomposición de las potencias presentes en los sistemas eléctricos. La figura 1 muestra la descomposición de las potencias para sistemas monofásicos del estándar IEEE 1459 (2010) y en el recuadro la descomposición de potencias propuesta para explicar los flujos de potencia ineficiente causados por el convertidor.

La cuantificación de potencia realizada por el estándar IEEE 1459 (2010) se resume en lo que sigue:

La potencia aparente efectiva $\left(S_{e}\right)$ cuantifica el conjunto de todas las potencias (eficientes e ineficientes que demanda la carga). Se divide en la potencia aparente efectiva fundamental $\left(S_{e 1}\right)$ y la potencia aparente efectiva no fundamental $\left(S_{e N}\right)$. 


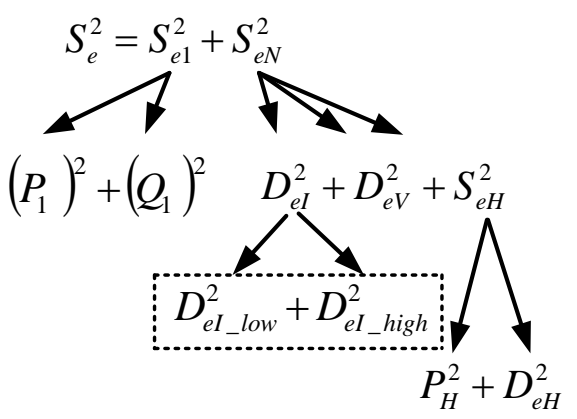

Fig 1. Potencias del estándar IEEE 1459 (2010) y en el recuadro términos de potencia propuestos.

$S_{e 1}$ cuantifica los fenómenos de potencia eléctrica provocados por las componentes fundamentales de voltaje y corriente. $S_{e 1}$ se divide en la potencia activa fundamental $\left(P_{1}\right)$ y en la potencia reactiva fundamental $\left(Q_{1}\right)$.

$P_{1}$ cuantifica la transferencia útil de potencia desde la fuente a la carga, por lo que es considerada la única potencia que es considera eficiente. $Q_{1}$ cuantifica los fenómenos de potencia eléctrica relacionados con el desfase de $I_{1}$ con respecto a $V_{1}$.

$S_{e N}$ cuantifica los fenómenos de potencia eléctrica provocados por componentes no fundamentales de voltaje y corriente. $S_{e N}$ se divide en la potencia de distorsión de corriente $\left(D_{e l}\right)$, en la potencia de distorsión de voltaje $\left(D_{e v}\right)$, y en la potencia aparente armónica $\left(S_{e H}\right)$.

$D_{e l}$ cuantifica los fenómenos de potencia eléctrica provocados por las corrientes no fundamentales.

$D_{e v}$ cuantifica los fenómenos de potencia eléctrica provocados por los voltajes no fundamentales.

$S_{e H}$ cuantifica los fenómenos de potencia eléctrica provocados por los voltajes y corrientes no fundamentales. $S_{e H}$ se divide en la potencia activa armónica $\left(P_{H}\right)$ y en la potencia aparente armónica residual $\left(D_{e H}\right)$.

De acuerdo a la teoría de la potencia instantánea Muñoz et al. (2011) y Seguí et al. (2007) y al estándar IEEE 1459 (2010), el único flujo de potencia eficiente de los sistemas monofásicos es $P_{1}$, el resto es ineficiente ya que no representan transferencia útil de potencia desde las fuentes hacia las cargas. En este artículo se realiza una descripción de los flujos de potencia provocados por corrientes armónicas generadas por el convertidor. Para esto se propone descomponer a la potencia $D_{e I}$ en la potencia de distorsión de corriente de baja frecuencia $\left(D_{\text {eI_low }}\right)$ y la potencia de distorsión de corriente de alta frecuencia $\left(D_{\text {eI_high }}\right)$, ver recuadro de la figura 1 . La potencia $D_{\text {eI_low }}$ reúne todos los flujos de potencia ineficientes causados por corrientes armónicas de baja frecuencia $\left(I_{h_{-} \text {low }}\right)$, en este artículo, corrientes cuyo orden $h$ es $\left(h_{-}\right.$low $=$ $3,5,7,9,11,13,15)$. La potencia $D_{\text {eI_high }}$ reúne todos los flujos de potencia ineficientes causados por corrientes armónicas de alta frecuencia $I_{h_{-} h i g h}$, en este artículo, corrientes cuyo orden $h$ es mayor o igual a 17 (h_high $\geq 17)$.

$$
\begin{aligned}
& D_{\text {eI_Low }}=V_{1} \cdot I_{h_{-} \text {low }}=V_{1} \cdot \sqrt{\sum_{h=h \_l o w} I_{h}^{2}} \\
& D_{\text {eI_High }}=V_{1} \cdot I_{h_{-} \text {high }}=V_{1} \cdot \sqrt{\sum_{h=h_{-} \text {high }} I_{h}^{2}}
\end{aligned}
$$

En el artículo no se estudian otros flujos de potencia causados por voltajes armónicos pues no tienden a propagarse por las redes de distribución o las cargas como lo hacen los flujos de potencia causados por corrientes armónicas. Además los convertidores tienen inductancias en su salida que absorben las tensiones armónicas permitiendo el acople del convertidor con la red de distribución.

\section{CONVERTIDOR DE POTENCIA Y MODULACIÓN SPWM}

El convertidor monofásico de la figura 2 está compuesto por un bus $\mathrm{DC}$, un puente $\mathrm{H}$ de dos ramas, y una carga AC. Cada rama contiene dos interruptores que conmutan de acuerdo a la estrategia de modulación. 
El disparo de los interruptores, en este caso IGBT's con diodos en antiparalelo, se realiza mediante las puertas G1, G2, G3, y G4. El convertidor convierte la tensión DC del bus de continua en tensión alterna AC inyectando las corrientes que requiere la carga.

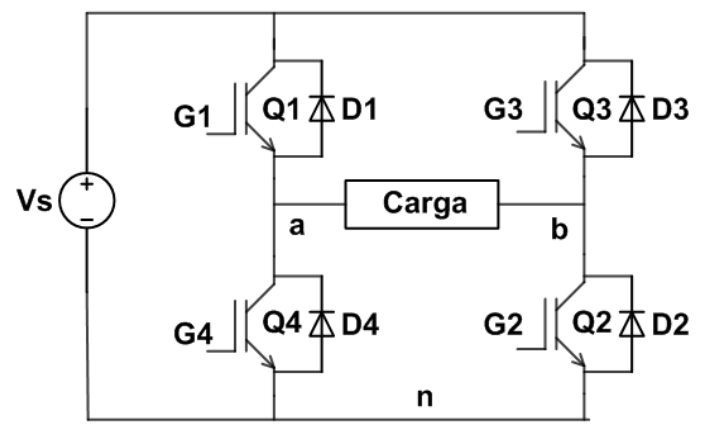

Fig 2. Convertidor topología puente $\mathrm{H}$

Todas las estrategias de modulación deben de seguir ciertas normas básicas de conmutación. Para evitar cortocircuitos, los interruptores de una misma rama no pueden estar cerrados simultáneamente. Los interruptores de una misma rama tampoco pueden estar abiertos simultáneamente pues se corre el riesgo de dejar bobinas abiertas con energía almacenada, esto podría ocasionar sobretensiones peligrosas en los interruptores que pueden dañar el inversor. De acuerdo a estas condiciones, existen tres posibilidades para el convertidor de la figura 2: 1) Interruptores Q1 y Q2 cerrados e interruptores Q3 y Q4 abiertos, en este caso el voltaje en la salida del convertidor $\left(V_{A B}\right)$ es positivo. 2) Interruptores $Q 1$ y $Q 2$ abiertos e interruptores Q3 y Q4 cerrados, en este caso $V_{A B}$ es negativo. 3) Interruptores Q2 y Q4 cerrados e interruptores Q1 y Q3 abiertos, en este caso $V_{A B}$ es cercano a cero. La corriente de la carga circula por los interruptores Q1, Q2, Q3, y Q4 cuando tiene el mismo sentido de la corriente marcada en el interruptor (hacia abajo), de lo contrario circula por los diodos D1, D2, D3, y D4.

Existen diferentes tipos de modulación para controlar el disparo de los interruptores, en este artículo se utiliza la estrategia de modulación SPWM. Los resultados obtenidos mediante la modulación SPWM pueden extrapolarse fácilmente para otras estrategias de modulación. La modulación SPWM consiste en comparar una señal de referencia (senoidal) con una señal portadora (triangular), ver figura 3. La comparación genera un tren de pulsos con ancho de pulso variables.

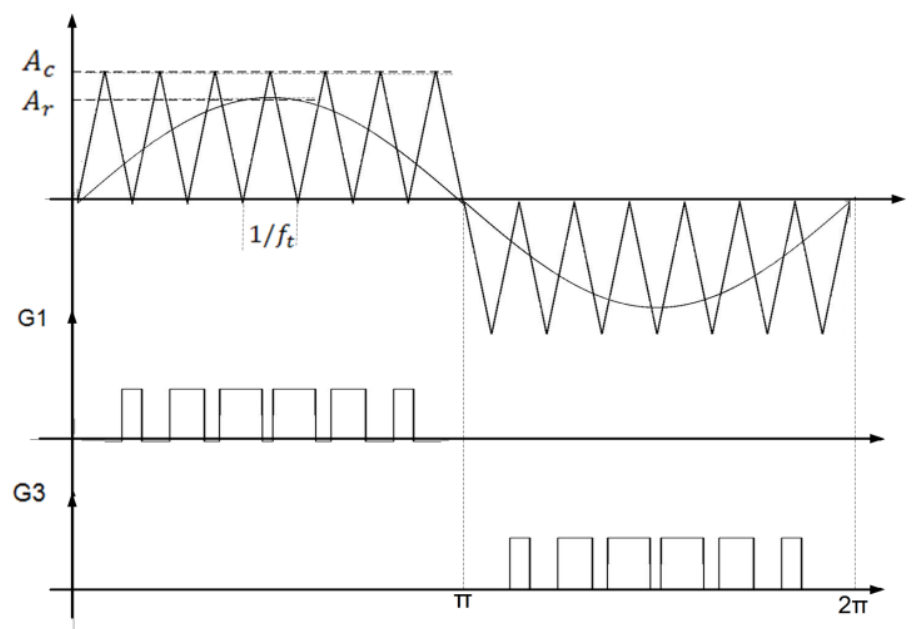

Fig 3. Generación de los pulsos para la modulación SPWM

La relación entre la amplitud de la señal de referencia $\left(A_{r}\right)$ y la señal portadora $\left(A_{c}\right)$ corresponde al índice de modulación $\left(m_{a}=A_{r} / A_{c}\right)$. El índice de modulación permite obtener una tensión variable a la salida del convertidor. La relación entre la frecuencia de la señal portadora $\left(f_{t}\right)$ y la frecuencia de la señal de referencia $\left(f_{r}\right)$ corresponde al índice de frecuencia $\left(m_{f}=2^{*}(n p+1)=f_{t} / f_{r}\right)$. Si el índice $m_{a}$ es menor a 1 , esta modulación elimina todos los armónicos de orden menor o igual a $\left(h<m_{f}-3\right)$. La generación de los pulsos de la modulación SPWM puede extenderse fácilmente a convertidores trifásicos, con la diferencia de que se deben poner 2 interruptores en una rama adicional (Para completar 3 fases). En este caso, las señales de cada fase deben estar desfasadas 120 grados para lograr un sistema trifásico de tensiones balanceadas en la salida del convertidor. El análisis de los flujos de potencia para sistemas trifásicos también sería similar incluso se podrían modelar por fase. 


\section{ANALISIS DE FLUJOS DE POTENCIA INEFICIENTES EN EL CONVERTIDOR}

En esta sección se describen de forma rigurosa los flujos de potencia en los convertidores causados por las caídas de tensión en los semiconductores de potencia. Para los IGBT's la caída de tensión entre colector y emisor, y para los diodos la caída de tensión entre ánodo y cátodo. Para el análisis se considera el convertidor de la figura 2 con carga resistiva-inductiva $(\mathrm{RL})$. La impedancia de los circuitos inductivos tiende a aumentar a medida que aumenta la frecuencia. Es por esto que el convertidor con carga $R L$ funciona como un filtro pasa bajas que reduce las componentes de alta frecuencia en la corriente, la magnitud de dichas corrientes depende significativamente de la estrategia de modulación usada en el convertidor. En la figura 4 se muestran la tensión, corriente y potencia de salida del convertidor para la modulación SPWM.

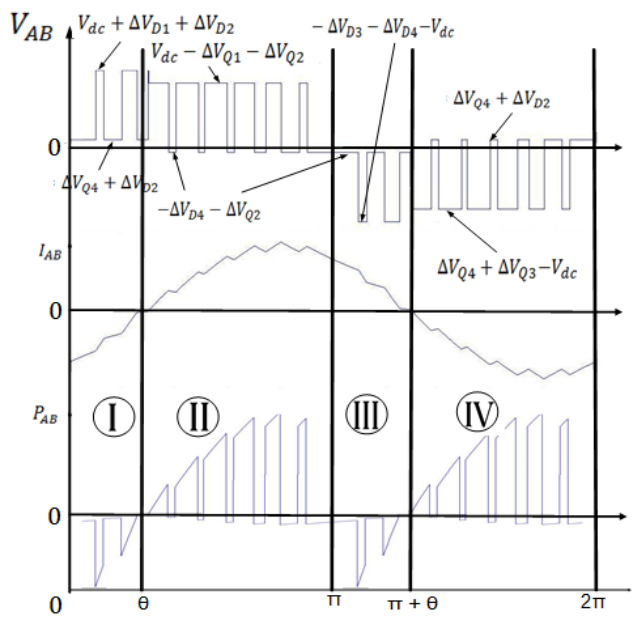

Fig 4. Tensión, corriente y potencia de salida del convertidor

El desfase $\theta$ (dado por $\theta=\tan ^{-1} \frac{\left|j \omega_{1} L\right|}{R} \operatorname{con} \omega_{1}$ la frecuencia angular fundamental de la onda de tensión y $L$ y $R$ los parámetros de la carga) entre el voltaje y la corriente establece 4 intervalos de conducción (I, II, II, IV), figura 4. Se analizarán estos intervalos en un periodo completo de tiempo tomando como referencia la tensión modulada de salida en el convertidor $\left(V_{A B}\right)$. Cada intervalo de conducción tiene dos posibles estados 1) Con tensión en la carga. 2) Sin tensión en la carga. Estos estados dependen de la posición de los interruptores del convertidor. En el intervalo (I) de conducción comprendido entre 0 y $\theta$, los dos posibles estados son: 1) Interruptores Q1 y Q2 cerrados e interruptores Q3 y Q4 abiertos. 2) Interruptores Q2 y Q4 cerrados e interruptores Q1 y Q3 abiertos. A continuación se describirán estos estados:

1) Interruptores Q1 y Q2 cerrados e interruptores Q3 y Q4 abiertos: En este estado de conducción, la carga está sometida a una tensión positiva $\left(V_{A B}>0\right)$. La corriente es negativa y de pendiente positiva disminuyendo su valor absoluto, esto indica que la bobina tiende a descargarse transfiriendo potencia a la fuente DC. En otras palabras la bobina de la carga tiene energía almacenada por lo que le entrega su energía a la fuente de DC a través de los diodos D1 y D2, esto se puede ver también en la curva de potencia que es negativa $\left(P_{A B}<0\right)$. Los diodos D3 y D4 no conducen. El voltaje $V_{A B}$ se puede calcular como sigue:

$$
V_{A B}=V_{A N}-V_{B N}=V_{d c}+\Delta V_{D 1}-\left(-\Delta V_{D 2}\right)=V_{d c}+\Delta V_{D 1}+\Delta V_{D 2}
$$

2) Interruptores Q2 y Q4 cerrados e interruptores Q1 y Q3 abiertos: Con respecto al estado anterior, los interruptores Q1 y Q4 cambiaron de estado mientras que los interruptores Q2 y Q3 permanecieron igual. La carga está sometida a una tensión cercana a cero debido a que Q2 y Q4 están cerrados. La corriente es negativa y de pendiente positiva disminuyendo su valor absoluto, esto indica que la bobina se descarga a través de las resistencias de los semiconductores Q4 y D2 y la resistencia de la carga, esto se puede ver también en la curva de potencia que es negativa y cercana a cero $\left(P_{A B} \approx 0\right)$. La fuente DC se encuentra abierta por lo que no intercambia energía con la carga. El voltaje en la carga puede calcularse como sigue:

$V_{A B}=V_{A N}-V_{B N}=\Delta V_{Q 4}-\left(-\Delta V_{D 2}\right)=\Delta V_{Q 4}+\Delta V_{D 2}$

En el intervalo (II) de conducción comprendido entre $\theta$ y $\pi$, los dos posibles estados son: 1) Interruptores Q1 y Q2 cerrados e interruptores Q3 y Q4 abiertos. 2) Interruptores Q2 y Q4 cerrados e interruptores Q1 y Q3 abiertos. A continuación se describirán estos estados: 
1) Interruptores $Q 1$ y $Q 2$ cerrados e interruptores $Q 3$ y $Q 4$ abiertos: En este estado de conducción, la carga está sometida a una tensión positiva $\left(V_{A B}>0\right)$. La corriente es positiva y de pendiente positiva aumentando su valor absoluto, esto indica que la bobina se está cargando. La fuente DC le entrega su energía almacenada a la bobina y lo hace a través de los interruptores Q1 y Q2, esto se puede ver también en la curva de potencia que es positiva $\left(P_{A B}>0\right)$. Ninguno de los diodos conduce por el sentido del flujo de la corriente. El voltaje en la carga está dado por:

$V_{A B}=V_{A N}-V_{B N}=V_{d c}-\Delta V_{Q 1}-\Delta V_{Q 2}$

2) Interruptores Q2 y Q4 cerrados e interruptores Q1 y Q3 abiertos: Con respecto al estado anterior, los interruptores Q1 y Q4 cambiaron de estado mientras que los interruptores Q2 y Q3 permanecieron igual. La carga está sometida a una tensión cercana a cero. debido a que Q2 y Q4 están cerrados. La corriente es positiva y de pendiente negativa disminuyendo su valor absoluto, esto indica que la bobina se está descargando transfiriendo su energía a los componentes resistivos de los semiconductores D4 y Q2 y la resistencia de la carga, esto se puede ver también en la curva de potencia que es negativa y cercana a cero $\left(P_{A B} \approx 0\right)$. La fuente DC se encuentra abierta por lo que no intercambia energía con la carga. El voltaje en la carga puede calcularse como sigue:

$V_{A B}=V_{A N}-V_{B N}=-\Delta V_{D 4}-\Delta V_{Q 2}$

En el intervalo (III) de conducción comprendido entre $\pi$ y $\pi+\theta$, los dos posibles estados son: 1) Interruptores Q3 y Q4 cerrados e interruptores Q1 y Q2 abiertos. 2) Interruptores Q2 y Q4 cerrados e interruptores Q1 y Q3 abiertos. A continuación se describirán estos estados:

1) Interruptores Q3 y Q4 cerrados e interruptores Q1 y Q2 abiertos: En este estado de conducción, la carga está sometida a una tensión negativa $\left(V_{A B}<0\right)$. La corriente circula en el convertidor a través de los diodos D3 y D4. Los diodos D1 y D2 no conducen. La corriente es positiva de pendiente negativa disminuyendo su valor absoluto, esto indica que la bobina se está descargando entregándole energía a la fuente $\mathrm{DC}\left(P_{A B}<0\right)$. El voltaje $V_{A B}$ se puede calcular como sigue:

$V_{A B}=V_{A N}-V_{B N}=-\Delta V_{D 4}-\left(V_{d c}+\Delta V_{D 3}\right)=-\Delta V_{D 3}-\Delta V_{D 4}-V_{d c}$

2) Interruptores Q2 y Q4 cerrados e interruptores Q1 y Q3 abiertos: Con respecto al estado anterior, los interruptores Q2 y Q3 cambiaron de estado mientras que los interruptores Q1 y Q4 permanecieron igual. La carga está sometida a una tensión cercana a cero voltios debido a que Q2 y Q4 están cerrados. La corriente es positiva de pendiente negativa disminuyendo su valor absoluto, esto indica que la bobina le transfiere energía a los componentes resistivos de los semiconductores D4 y Q2 y a la resistencia de la carga $\left(P_{A B} \approx\right.$ 0 , negativa cercana a cero). La fuente DC se encuentra abierta por lo que no intercambia energía con la carga. El voltaje en la carga puede calcularse como sigue:

$V_{A B}=-\Delta V_{D 4}-\Delta V_{Q 2}$

En el intervalo (IV) de conducción comprendido entre $\pi+\theta$ y $2 \pi$, los dos posibles estados son: 1) Interruptores Q3 y Q4 cerrados e interruptores Q1 y Q2 abiertos. 2) Interruptores Q2 y Q4 cerrados e interruptores Q1 y Q3 abiertos. A continuación se describirán estos estados:

1) Interruptores Q3 y Q4 cerrados e interruptores Q1 y Q2 abiertos: En este estado de conducción, la carga está sometida a una tensión negativa $\left(V_{A B}<0\right)$. La corriente circula en el convertidor a través de los interruptores Q3 y Q4. Ninguno de los diodos conduce por el sentido del flujo de la corriente. La corriente es negativa de pendiente negativa aumentando su valor absoluto, esto indica que la fuente DC está cediendo energía y le está transfiriendo energía a la bobina que se está cargando $\left(P_{A B}>0\right)$. El voltaje en la carga está dado por:

$V_{A B}=V_{A N}-V_{B N}=\Delta V_{Q 4}-\left(V_{d c}-\Delta V_{Q 3}\right)=\Delta V_{Q 4}+\Delta V_{Q 3}-V_{d c}$

2) Interruptores Q2 y Q4 cerrados e interruptores Q1 y Q3 abiertos: Con respecto al estado anterior, los interruptores Q2 y Q3 cambiaron de estado mientras que los interruptores Q1 y Q4 permanecieron igual. La carga está sometida a una tensión cercana a cero voltios debido a que Q2 y Q4 están cerrados. La corriente es negativa de pendiente positiva disminuyendo su valor absoluto, esto indica que la bobina le está transfiriendo energía a los componentes resistivos de los semiconductores Q4 y D2 y a la resistencia de la carga $\left(P_{A B} \approx 0\right.$, negativa cercana a cero). La fuente DC se encuentra abierto por lo que no intercambia energía con la carga. El voltaje en la carga puede calcularse como sigue: 
$V_{A B}=V_{A N}-V_{B N}=\Delta V_{Q 4}-\left(-\Delta V_{D 2}\right)=\Delta V_{Q 4}+\Delta V_{D 2}$

En la figura 3 se muestra la forma de onda de la tensión de salida del convertidor para los cuatro intervalos de conducción. En el caso ideal, la tensión de salida del convertidor varía entre $V_{d c}$, cero y $-V_{d c}$. Mientras que en el caso real, la tensión de salida está dada por las ecuaciones (3-10). Las caídas de tensión en los semiconductores provocan la deformación de la tensión de salida del convertidor provocando armónicos de tensión y de corriente.

\section{RESULTADOS DE SIMULACIÓN}

A continuación se presentan los resultados de simulación de un convertidor con topología puente $\mathrm{H}$. Se utilizó Matlab-Simulink para realizar las simulaciones, el circuito implementado puede verse en la figura 4. Para generar las señales de la modulación SPWM (G1, G2, G3, G4) se utilizó la herramienta S-Function de Simulink. Una S-Function permite crear bloques en Simulink mediante código de matlab utilizando archivos de programación "M".

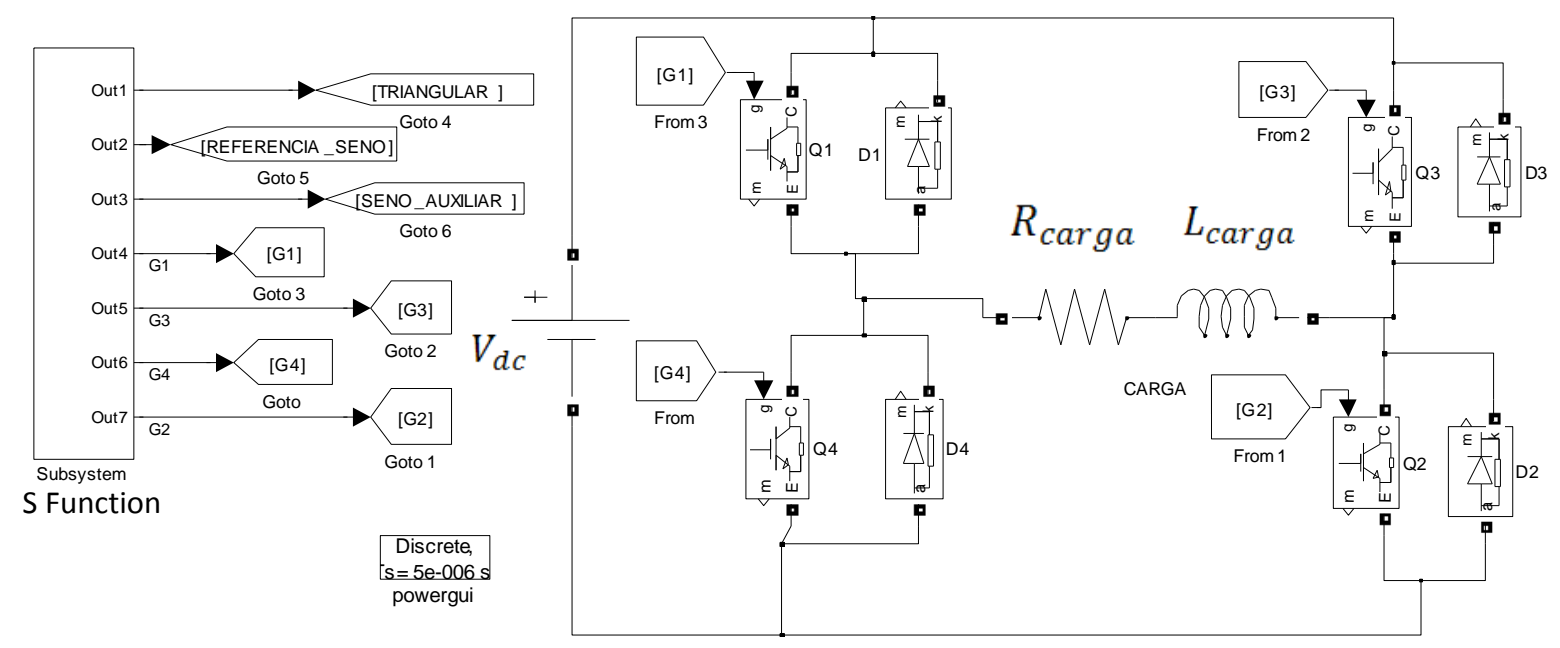

Fig 5. Simulación topología puente H Matlab-Simulink

Para la simulación se consideran dos análisis: 1) Análisis de los flujos de potencia en función de la caída de tensión en los interruptores de potencia del convertidor. 2) Análisis de los flujos de potencia del convertidor en función del número de pulsos de la estrategia de modulación.

Análisis de los flujos de potencia del convertidor cuando varía la caída de tensión ( $\Delta V)$ de los interruptores de potencia.

Este análisis considera la topología de la figura 4, en la cual se utilizan los siguientes valores para realizar la simulación: voltaje en el bus de continua $\left(V_{d c}=100 \mathrm{~V}\right)$, resistencia en la carga $\left(R_{\text {carga }}=3.6 \Omega\right)$, inductancia en la carga $\left(L_{\text {carga }}=5.5 \mathrm{mH}\right)$, frecuencia fundamental de salida del convertidor $\left(f_{1}=50 \mathrm{~Hz}\right)$, índice de modulación $\left(m_{a}=0.8\right)$, y número de pulsos de la modulación $(n p=20)$. La caída de tensión $\Delta V$ varía desde el caso ideal $0 \mathrm{~V}$ hasta $1.8 \mathrm{~V}$. (En las hojas de datos de los interruptores de potencia se observa que la mayoría presentan $\Delta V$ entre 0.5 y $1.8 \mathrm{~V}$ ).

La tabla 1 muestra el espectro de corrientes en la salida del convertidor para diferentes caídas de tensión en los semiconductores de potencia. En el caso ideal, cuando la caída de tensión de los semiconductores es cero, los armónicos de corriente de bajo orden $(n=3,5,7,9,11,13,15)$ son despreciables tendiendo a cero. A medida que $\Delta V$ aumenta, estos armónicos aumentan de valor. La corriente fundamental $I_{1}$ disminuye a medida que aumenta $\Delta V$. Los armónicos de corriente de alto orden $\left(h \_h i g h=39,41,43,45,47,49\right)$ permanecen constantes a medida que aumenta $\Delta V$.

La caída de tensión de los semiconductores del inversor provoca flujos de potencia ineficientes. En la tabla 2 , se muestra la evolución del voltaje, corrientes y potencias en la salida del convertidor cuando se aumenta la caída de tensión en los semiconductores de potencia. 
Tabla 1. Espectro de corriente en función de $\Delta V$.

\begin{tabular}{|c|r|r|r|r|r|r|r|r|r|r|}
\hline$\Delta V[V]$ & 0 & 0.2 & 0.4 & 0.6 & 0.8 & 1 & 1.2 & 1.4 & 1.6 & 1.8 \\
\hline$I_{1}$ & 20.024 & 19.909 & 19.794 & 19.679 & 19.563 & 19.448 & 19.333 & 19.217 & 19.102 & 18.986 \\
\hline$I_{3}$ & 0.003 & 0.024 & 0.051 & 0.078 & 0.105 & 0.131 & 0.158 & 0.185 & 0.212 & 0.239 \\
\hline$I_{5}$ & 0.003 & 0.011 & 0.022 & 0.033 & 0.044 & 0.055 & 0.066 & 0.077 & 0.088 & 0.098 \\
\hline$I_{7}$ & 0.003 & 0.003 & 0.009 & 0.014 & 0.020 & 0.026 & 0.032 & 0.037 & 0.043 & 0.049 \\
\hline$I_{9}$ & 0.001 & 0.004 & 0.008 & 0.011 & 0.015 & 0.018 & 0.022 & 0.026 & 0.029 & 0.033 \\
\hline$I_{11}$ & 0.001 & 0.002 & 0.005 & 0.007 & 0.009 & 0.012 & 0.014 & 0.017 & 0.019 & 0.022 \\
\hline$I_{13}$ & 0.001 & 0.002 & 0.003 & 0.005 & 0.007 & 0.008 & 0.010 & 0.012 & 0.014 & 0.015 \\
\hline$I_{15}$ & 0.002 & 0.001 & 0.002 & 0.003 & 0.004 & 0.006 & 0.007 & 0.008 & 0.009 & 0.011 \\
\hline$I_{39}$ & 0.206 & 0.206 & 0.206 & 0.206 & 0.206 & 0.206 & 0.206 & 0.206 & 0.206 & 0.205 \\
\hline$I_{41}$ & 0.443 & 0.443 & 0.443 & 0.443 & 0.443 & 0.443 & 0.443 & 0.443 & 0.443 & 0.443 \\
\hline$I_{43}$ & 0.423 & 0.423 & 0.423 & 0.423 & 0.422 & 0.422 & 0.422 & 0.422 & 0.422 & 0.422 \\
\hline$I_{45}$ & 0.179 & 0.179 & 0.179 & 0.179 & 0.179 & 0.179 & 0.179 & 0.178 & 0.178 & 0.178 \\
\hline$I_{47}$ & 0.015 & 0.015 & 0.015 & 0.015 & 0.015 & 0.015 & 0.015 & 0.015 & 0.015 & 0.015 \\
\hline$I_{49}$ & 0.000 & 0.001 & 0.001 & 0.001 & 0.001 & 0.001 & 0.001 & 0.001 & 0.001 & 0.001 \\
\hline
\end{tabular}

La tensión fundamental $V_{1}$ disminuye a medida que aumenta $\Delta V$. La corriente $I_{h_{-} \text {low }}$ aumenta debido a que las corrientes armónicas de bajo orden aumentan. La corriente $I_{h_{-} h i g h}$ permanece igual porque las corrientes armónicas de alto orden no cambian significativamente. La disminución de $V_{1}$ e $I_{1}$ provocan la reducción de $P_{1}$ y $Q_{1}$. El aumento $I_{h_{-} \text {low }}$ provoca el aumento de $D_{\text {eI_low }}$. La potencia $D_{\text {eI_high }}$ permanece constante pues $I_{h \_ \text {high }}$ no cambia. El aumento de $\Delta V$ provoca la disminución de la potencia $P_{1}$ eficiente que suministra el convertidor y el aumento de la potencia $D_{\text {eI_low }}$ considerada ineficiente. El flujo ineficiente $D_{\text {eI_low }}$ depende significativamente de la caída de tensión de los semiconductores de los interruptores de potencia, por lo que se deben elegir interruptores con bajas caídas de tensión de tal forma que se aproveche al máximo la potencia del convertidor.

Tabla 2. Tensiones, corrientes, potencias del convertidor en función de $\Delta V$.

\begin{tabular}{|c|r|r|r|r|r|r|r|r|r|r|}
\hline$\Delta V[V]$ & 0 & 0.2 & 0.4 & 0.6 & 0.8 & 1 & 1.2 & 1.4 & 1.6 & 1.8 \\
\hline$V_{1}[\mathrm{~V}]$ & 80.0 & 79.5 & 79.0 & 78.6 & 78.1 & 77.7 & 77.2 & 76.7 & 76.3 & 75.8 \\
\hline$I_{\text {hlow }}[\mathrm{A}]$ & 0.006 & 0.027 & 0.057 & 0.087 & 0.117 & 0.147 & 0.177 & 0.207 & 0.237 & 0.267 \\
\hline$I_{h_{\text {high }}[\mathrm{A}]}$ & 0.671 & 0.671 & 0.671 & 0.671 & 0.671 & 0.670 & 0.670 & 0.670 & 0.670 & 0.670 \\
\hline$P_{1}[\mathrm{~W}]$ & 1441.0 & 1424.5 & 1408.1 & 1391.7 & 1375.5 & 1359.3 & 1343.2 & 1327.2 & 1311.3 & 1295.5 \\
\hline$Q_{1}[\mathrm{var}]$ & 697.9 & 689.9 & 682.0 & 674.0 & 666.2 & 658.3 & 650.5 & 642.8 & 635.1 & 627.4 \\
\hline$D_{\text {eI_low }}[\mathrm{va}]$ & 0.447 & 2.157 & 4.495 & 6.816 & 9.118 & 11.391 & 13.636 & 15.855 & 18.039 & 20.201 \\
\hline$D_{\text {eI_high }}[\mathrm{va}]$ & 53.636 & 53.329 & 53.013 & 52.701 & 52.383 & 52.062 & 51.739 & 51.416 & 51.093 & 50.768 \\
\hline
\end{tabular}

Análisis de los flujos de potencia del convertidor cuando varía el número de pulsos de la estrategia de modulación.

Este análisis considera la topología de la figura 4, en la cual se utilizan los siguientes valores para realizar la simulación: voltaje en el bus de continua $\left(V_{d c}=100 \mathrm{~V}\right)$, resistencia en la carga $\left(R_{\text {carga }}=3.6 \Omega\right)$, inductancia en la carga $\left(L_{\text {carga }}=5.5 \mathrm{mH}\right)$, frecuencia fundamental de salida del convertidor $\left(f_{1}=50 \mathrm{~Hz}\right)$, índice de modulación $\left(m_{a}=0.8\right)$, caída de tensión en los semiconductores de potencia $(\Delta V=1 \mathrm{~V})$.

La tabla 3 muestra el espectro de corrientes en la salida del convertidor cuando cambian el número de pulsos de la estrategia de modulación. La corriente fundamental $I_{1}$ y las corrientes armónicas de bajo orden $(h=3,5,7,9,11,13,15)$ permanecen constantes no dependiendo del número de pulsos elegidos en la estrategia de modulación. Las corrientes armónicas de alto orden ( $\left.h \_h i g h \geq m_{f}-3\right)$ disminuyen a medida que aumenta el número de pulsos. Las corrientes armónicas de alto orden y que aparecen en el espectro siguen la ecuación $\left(h_{\text {high }}+2 \cdot k\right.$ con $\left.k=0,1,2,3,4,5\right)$ desplazándose hacia la derecha del espectro a medida que aumenta $m_{f}$.

En la tabla 4, se muestra la evolución del voltaje, corrientes y potencias en la salida del convertidor cuando se aumenta el número de pulsos de la estrategia de modulación. La tensión fundamental $V_{1}$ y la corriente $I_{h_{-} \text {low }}$ permanecen prácticamente constantes. La corriente $I_{h \_ \text {high }}$ disminuye cuando el número de pulsos aumenta. Las potencias $P_{1}, Q_{1}$, y $D_{\text {eI_low }}$ permanecen constantes no dependiendo del número de pulsos del convertidor. Mientras que la potencia $D_{\text {el_high }}$ disminuye a medida que aumenta el número de pulsos de la estrategia de modulación. 
Tabla 3. Espectro de corriente en función del número de pulsos $(n p)$.

\begin{tabular}{|c|r|r|r|r|r|r|r|r|r|r|}
\hline$n p$ & 10 & 20 & 30 & 40 & 50 & 60 & 70 & 80 & 90 & 100 \\
\hline$I_{1}$ & 19.463 & 19.448 & 19.448 & 19.448 & 19.449 & 19.442 & 19.439 & 19.457 & 19.446 & 19.457 \\
\hline$I_{3}$ & 0.135 & 0.131 & 0.136 & 0.139 & 0.137 & 0.135 & 0.134 & 0.136 & 0.135 & 0.134 \\
\hline$I_{5}$ & 0.053 & 0.055 & 0.055 & 0.056 & 0.055 & 0.054 & 0.051 & 0.055 & 0.057 & 0.054 \\
\hline$I_{7}$ & 0.030 & 0.026 & 0.029 & 0.025 & 0.028 & 0.025 & 0.025 & 0.029 & 0.025 & 0.032 \\
\hline$I_{9}$ & 0.018 & 0.018 & 0.017 & 0.017 & 0.017 & 0.017 & 0.015 & 0.017 & 0.018 & 0.017 \\
\hline$I_{11}$ & 0.011 & 0.012 & 0.013 & 0.012 & 0.011 & 0.013 & 0.012 & 0.012 & 0.012 & 0.012 \\
\hline$I_{13}$ & 0.010 & 0.008 & 0.009 & 0.008 & 0.008 & 0.008 & 0.008 & 0.009 & 0.008 & 0.006 \\
\hline$I_{15}$ & 0.007 & 0.006 & 0.006 & 0.007 & 0.006 & 0.008 & 0.006 & 0.007 & 0.009 & 0.007 \\
\hline$I_{h_{\_} \text {high }}$ & 0.418 & 0.206 & 0.137 & 0.102 & 0.081 & 0.068 & 0.058 & 0.051 & 0.045 & 0.041 \\
\hline$I_{h_{\_} \text {high }+2}$ & 0.860 & 0.443 & 0.298 & 0.224 & 0.180 & 0.150 & 0.129 & 0.113 & 0.100 & 0.090 \\
\hline$I_{h_{\text {_high }}+4}$ & 0.788 & 0.422 & 0.289 & 0.219 & 0.177 & 0.148 & 0.127 & 0.111 & 0.100 & 0.090 \\
\hline$I_{h_{\text {_high }}+6}$ & 0.320 & 0.179 & 0.124 & 0.095 & 0.077 & 0.065 & 0.056 & 0.049 & 0.044 & 0.039 \\
\hline$I_{h_{\_} \text {high+8 }}$ & 0.026 & 0.015 & 0.011 & 0.008 & 0.007 & 0.006 & 0.005 & 0.004 & 0.004 & 0.004 \\
\hline$I_{h_{\_} \text {high }+10}$ & 0.002 & 0.001 & 0.001 & 0.000 & 0.000 & 0.000 & 0.000 & 0.000 & 0.000 & 0.000 \\
\hline
\end{tabular}

Tabla 4. Tensiones, corrientes, potencias del convertidor en función de $n p$.

\begin{tabular}{|c|r|r|r|r|r|r|r|r|r|r|}
\hline$n p$ & 10 & 20 & 30 & 40 & 50 & 60 & 70 & 80 & 90 & 100 \\
\hline$V_{1}[\mathrm{~V}]$ & 77.72 & 77.66 & 77.66 & 77.66 & 77.67 & 77.64 & 77.62 & 77.70 & 77.65 & 77.69 \\
\hline$I_{\text {h_low }}[A]$ & 0.150 & 0.147 & 0.151 & 0.154 & 0.152 & 0.150 & 0.148 & 0.151 & 0.150 & 0.149 \\
\hline$I_{\text {h_high }}[A]$ & 1.280 & 0.670 & 0.454 & 0.343 & 0.276 & 0.231 & 0.198 & 0.174 & 0.155 & 0.139 \\
\hline$P_{1}[\mathrm{~W}]$ & 1361.4 & 1359.3 & 1359.2 & 1359.3 & 1359.5 & 1358.5 & 1358.0 & 1360.6 & 1359.0 & 1360.5 \\
\hline$Q_{1}[\mathrm{var}]$ & 659.3 & 658.3 & 658.3 & 658.4 & 658.4 & 657.9 & 657.7 & 659.0 & 658.2 & 658.9 \\
\hline$D_{\text {eI_low }}[\mathrm{va}]$ & 11.628 & 11.391 & 11.735 & 11.928 & 11.769 & 11.605 & 11.450 & 11.755 & 11.655 & 11.595 \\
\hline$D_{\text {eI_high }}[\mathrm{va}]$ & 99.476 & 52.062 & 35.270 & 26.669 & 21.431 & 17.916 & 15.379 & 13.493 & 12.014 & 10.821 \\
\hline
\end{tabular}

La figura 5 muestra la variación de $D_{\text {el_high }}$ con respecto al número de pulsos del convertidor. A medida que aumentan los pulsos, la potencia $D_{\text {eI_high }}$ disminuye. La pendiente de la curva tiende a disminuir a medida que aumenta el número de pulsos. Cuando la pendiente tiende a cero, el aumento del número de pulsos no provoca una reducción significativa de $D_{\text {el_high. }}$. Aumentar el número de pulsos de 80 a 90 , por ejemplo, solo provoca la reducción de $D_{\text {el_high }}$ de $13.49 \mathrm{va}$ a $12.01 \mathrm{va}$. El aumento del número de pulsos reduce $D_{\text {eI_high }}$ pero también provoca el aumento de la frecuencia de conmutación. El aumento de la frecuencia ocasiona el aumento de las pérdidas por conmutación, es por eso que no se deben incrementar los pulsos excesivamente Huang et al. (2010). Los diseñadores de inversores pueden tomar a $D_{\text {eI_high }}$ como criterio para establecer el número de pulsos efectivos reduciendo los flujos de potencia ineficientes y aumentando la eficiencia del convertidor. Un análisis del número de pulsos efectivos para convertidores de potencia teniendo en cuenta a $D_{\text {eI_high }}$ y las pérdidas por conmutación será presentado en futuras publicaciones.

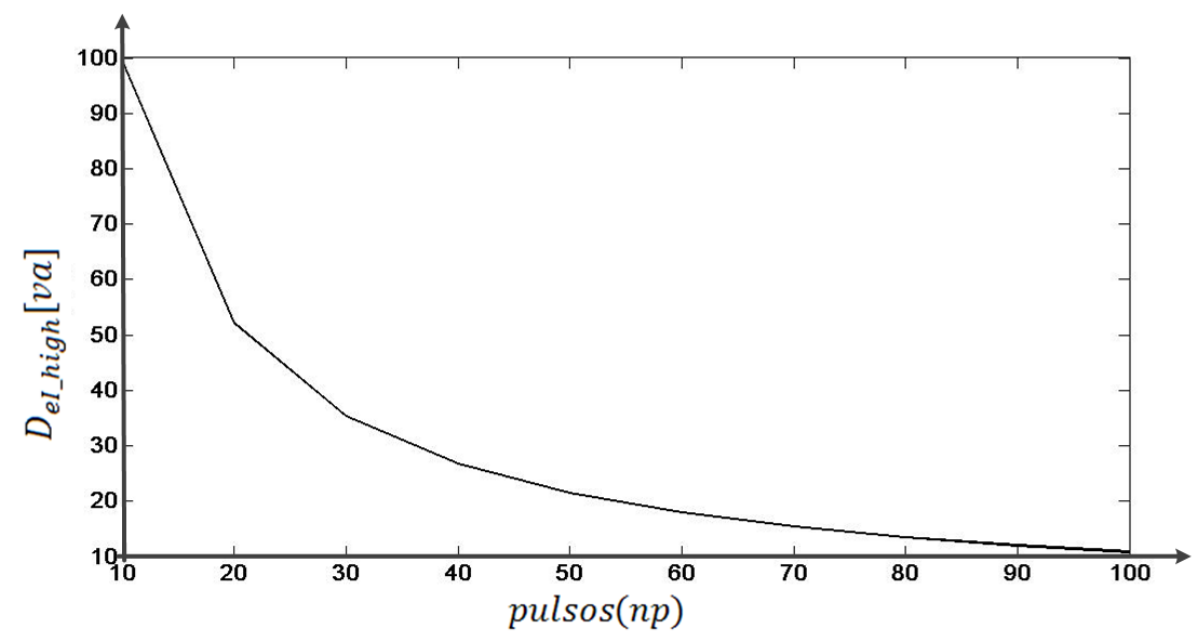

Fig 6. Potencia $D_{\text {eI_high }}$ en función de $n p$ 


\section{CONCLUSIONES}

El artículo presenta un análisis de los flujos de potencia ineficientes provocados por los convertidores de potencia. Se identificaron dos causas:

1) Las caídas de tensión en los semiconductores de los interruptores de potencia provocan armónicos de baja frecuencia $(h=3,5,7,9,11,13,15)$, al aumentar las caídas de tensión aumenta el contenido armónico. Para cuantificar este fenómeno se utilizó la potencia $D_{\text {el_low }}$ que aumenta a medida que aumenta la caída de tensión en los interruptores del convertidor. Cuando se trabaja la modulación SPWM con un índice de modulación $m_{a}$ menor a 1, la teoría indica que los armónicos de baja frecuencia no aparecen en el espectro, sin embargo aparecen debido a las caídas de tensión en los semiconductores de los interruptores de potencia. Aumentar las caídas de tensión también reduce significativamente la potencia $P_{1}$ (eficiente) que es capaz de entregar el convertidor.

2) La amplitud de los armónicos de alta frecuencia $\left(h \_h i g h \geq m_{f}-3\right)$ disminuye a medida que aumenta el índice de frecuencia $m_{f}$. Para cuantificar este fenómeno se utilizó la potencia $D_{\text {eI_high }}$ que disminuye a medida que $m_{f}$ aumenta. El flujo de potencia $D_{\text {eI_high }}$ debe reducirse para mejorar la calidad de la potencia en la red de distribución evitando que corrientes armónicas de alta frecuencia circulen hacia la red. La potencia $D_{\text {eI_high }}$ puede utilizarse desde la etapa de diseño para diseñar convertidores más eficientes con estándares altos en calidad de la potencia. La medida de $D_{\text {eI_high }}$ puede servir para diagnosticar problemas de calidad de la potencia asociados a la inyección de corrientes de alta frecuencia, además puede servir para establecer un criterio para penalizar a las empresas que inyecten a la red corrientes de alta frecuencia.

\section{AGRADECIMIENTOS}

Los autores agradecen a la Universidad de Antioquia (UdeA) por el apoyo de "Sostenibilidad 2013-2014". Igualmente se agradece al CODI de la UdeA por financiar el proyecto "Diseño y construcción de Prototipo Convertidor Trifásico DC-AC".

\section{REFERENCIAS}

Huang, R., y Mazumder, S. K., A Soft Switching Scheme for Multiphase DC/Pulsating-DC Converter for Three-Phase High-Frequency-Link Pulsewidth Modulation (PWM) Inverter. IEEE Transactions on Power Electronics, 25(7), 1761-1774, (2010).

IEEE Std. 1459, IEEE Trial Use Standard Definitions for the Measurement of Electric Power Quantities under Sinusoidal, Non-Sinusoidal, Balanced, or Unbalanced Conditions.. Ins. of Electrical and Electronics Engineers, (2010).

Jacobina, C. B., dos Santos, E. C., Rocha, N., y Lopes Fabricio, E. L., Single-Phase to Three-Phase Drive System Using Two Parallel Single-Phase Rectifiers. IEEE Transactions on Power Electronics, 25(5), 12851295, (2010).

Kim, H., F. Blaabjert, y B. Bak-Jensen, Spectral Analysis of Instantaneous Powers in Single-Phase and Three-Phase Systems with Use of p-qr Theory, IEEE Trans. Power Electronics, 17 (5), 711-720, (2002).

Kojabadi, H. M., A comparative analysis of different pulse width modulation methods for low cost induction motor drives. Energy Conversion and Management, 52(1), 136-146, (2011).

Komurcugil, H., Rotating-Sliding-Line-Based Sliding-Mode Control for Single-Phase UPS Inverters. IEEE Transactions on Industrial Electronics, 59(10), 3719-3726, (2012).

Munoz-Galeano N. y otros 4 autores, Non-fundamental effective apparent power defined through an instantaneous power approach, Int. J. Electrical Power and Energy Systems. 33 (10), 1711-1720, (2011).

Orts, S., A. Abellán, F. Gimeno, S. Seguí, y J. Alfonso, Improved Shunt Active Power Compensator for IEEE Standard 1459 Compliance, IEEE Trans. Power Delivery, 25 (4), 2692-2701, (2010).

Rus, D. C., Preda, N. S., Incze, I. I., Imecs, M., y Szabó, C., Comparative analysis of PWM techniques: Simulation and DSP implementation. IEEE International Conference on Automation Quality and Testing Robotics (AQTR) 3(1), 1-6, (2010).

Seguí-Chilet S. y otros 6 autores, Approach to unbalance power active compensation under linear load unbalances and fundamental voltage asymmetries, Int. J. Electrical Power and Energy Systems. 29 (7), 526539, (2007). 